\title{
The Severity of Polluted Insulator Surface Based on the Leakage Current Harmonic Measurements
}

\author{
Carlos RS ${ }^{1,3}$ Zainuddin Nawawi $^{2}$ Irfan Jambak $^{2}$ Rumiasih $^{1}$ Anton Firmansyah ${ }^{1}$ \\ Indah Susanti ${ }^{1}$ \\ ${ }^{1}$ Electrical Engineering Department, State Polytechnic of Sriwijaya, Jalan Srijaya Negara, Palembang, 30139 \\ Indonesia \\ ${ }^{2}$ Electrical Engineering Department, Sriwijaya University, Jalan Palembang-Inderalaya, Indonesia \\ ${ }^{3}$ Engineering Department of Doctoral Degree, Sriwijaya University, Jalan Palembang-Inderalaya, Indonesia \\ *Corresponding author. Email: carlos@polsri.ac.id
}

\begin{abstract}
One of the important components in the high voltage overhead lines placed on the transmission tower is an insulator. Insulators that are installed outdoor will be exposed to the environment directly. Due to environmental conditions and pollutants attached to the surface, leakage currents can flow on the surface of the insulator. Large leakage currents can damage the surface of the insulator and cause losses in the form of heat and even cause flashover. This paper provides an alternative way to prevent early flashover by detecting the severity of the insulator surface based on harmonic measurements of leakage currents. Insulator performance mainly depends on the conductivity of the surface layer being polluted or by generating pollutants via the equivalent salt deposit density (ESDD). The leakage currents were evaluated at different ESDD levels as deposits of very light, light, moderate, and heavy $\mathrm{NaCl}$ salt pollution on a $20 \mathrm{kV}$ outdoor polymer insulators. From the experiments, it can be concluded that: i. The leakage current that occurs when the surface of the insulator is very lightly polluted has an unsymmetrical waveform that is distorted and leads to a positive with a large THD; ii. The magnitude of the leakage current with the surface of the insulator is polluted, which leads to greater weight, but the harmonic distortion and THD are getting smaller with the waveform of the leakage current signal leading to a sinusoidal waveform.
\end{abstract}

\section{Keywords: Insulator surface, harmonic measurements, Leakage current}

\section{INTRODUCTION}

For transmitting a large amounts of power from power plant to the load centre in long distance, a transmission line is needed. For overhead line, the highvoltage or extra-high voltage transmission lines are usually used. Insulator is one of the important things in the high voltage overhead lines. This insulator is usually placed on the towers or concrete poles. It main function is for supporting the conductive wire and as the insulator of one voltage part to the other voltage parts with different phases or to the non-voltage conductor part on the tower or concrete pole overhead lines.

In general, the building of high voltage overhead transmission lines is much more easy in the coastal areas on the grounds due to these areas are already developed before. In rejuvenating and constructing good transmission lines, it is very important to pay more attention on the design of the tower and the insulators' type. The insulators should be non-conductive to electric current. All of the high-voltage overhead transmission lines insulators are outdoors type which properties change easily due to the environment condition, such as pollution.
Most of the insulators used for the overhead lines, either medium, high, or extra high voltage in Indonesia are made of ceramic and glass. However, these materials have some drawbacks, such as: the large mass density that they have and the big losses that they serve. Thus, it leads to the construction and maintenance of the power grid.

To overcome the problems that are occurred by the glass and ceramic insulator, many researchers presented polymer insulators made of silicon rubber (SIR) and ethylene-propylene-diene monomer (EPDM). These former materials have several advantages, such as: they have light weight so that they are easy to be moved, transported, and installed. In addition, they have high mechanical strength and aesthetic appearance. Besides that, silicon rubber polymer insulators have high resistance to surface wetting (hydrophobic), so that it can provide high electrical surface resistance [1]. However, the hydrophobicity of polymer insulators decreases with long-term aging of the material due to environmental stresses or area of use.

Installing the insulators in Indonesia has some challenges that should be paid attention. It is due to 
Indonesia is included as one of the countries experiencing the highest levels of pollution in the world. When polymer insulators are installed in tropical climates, pollutant particles that are sticked to the surface of the insulators cause the occurrence of impurities. This condition will be worse in the wet season where the leakage current will flow to the ground trough the grounding system due to the conductive layer that has been formed by the impurities on the surface of the insulator [2]. This situation can lead to the occurrence of the dry bands on the surface of the insulator, in which at the end can also cause the electric arc or Partial Discharge (PD).

The dry bands will not only cause the erosion but also the degradation performance of the insulator surface. The flashover [3] will occur as the result of the dry band arcs that occur along the profile of the insulator. When this condition happens, the transmission line will be go out and can cause the disruption and the decreasing on the electrical power system. The flashover, actually can be hint by cleaning the surface of the insulator regularly, however, it is difficult to do and of course it needs additional cost.

The performance of the insulator depends on the conductivity of the polluted surface layer or equivalent salt deposit density (ESDD) [4]. ESDD can be used to determine the resistance of salt deposits as a parameter in the developed equivalent circuit model. This study provides an alternative way to prevent early flashover by detecting the condition of the surface severity of the insulator based on harmonic measurements of leakage currents. In this study, the equivalent circuit model was tested to measure the leakage current signal. The leakage current signal based on the harmonic frequency spectrum was evaluated on the different surface severity of the insulator, i.e., from the salt pollution in the $20 \mathrm{kV}$ outdoor polymer insulator. The leakage current signal based on the harmonic frequency spectrum is evaluated based on the severity of the surface of the insulator in order to: i) know the magnitude of the leakage current signal that occurs as an indication of the occurrence of contamination on the surface of the polymer insulator, ii) know the frequency spectrum of the leakage current signal for the level of contamination on the surface of the insulator, that is classified into 4 groups, namely: very light, light, moderate, and heavy. iii) to determine the total harmonic distortion for those 4 groups insulator surface contamination levels (very light, light, moderate, and heavy).

\section{RESEARCH METHOD}

The data in this research was obtained by measuring the leakage current in the insulator with the Simulink model using Matlab software. The simulation model uses an equivalent circuit of suspension insulators from silicon rubber material which is placed on a transmission tower. The pollution resistance can be calculated with the help of the conductivity of the pollution. To obtain the leakage current pattern at the very light, light, moderate, and severe pollution severity is determined based on the ESDD according to the IEEE 4-1995 standard. The equivalent salt deposit density is used to determine the pollution resistance ( $\mathrm{Rp}$ ) on the insulator surface. The harmonic pattern of the leakage current is measured based on the severity of the pollution through the frequency spectrum of the leakage current to determine the harmonic content of the leakage current in total harmonic distortion (THD).

Measurement of leakage current on the surface of polluted polymer insulators is obtained by modeling using Matlab software and a personal computer. The high voltage test model is made in Matlab Simulink with several components of the peak-to-peak voltage $\left(V_{p}\right)$ supply, the series resistance $\left(R_{s}\right)$ as the voltage supply resistance, the shunt capacitor as the insulator's capacitance to the ground, the insulator capacitance, the pollution resistance according to the pollution level, the current instrument, and scope to display measurement results as shown in Figure 1.

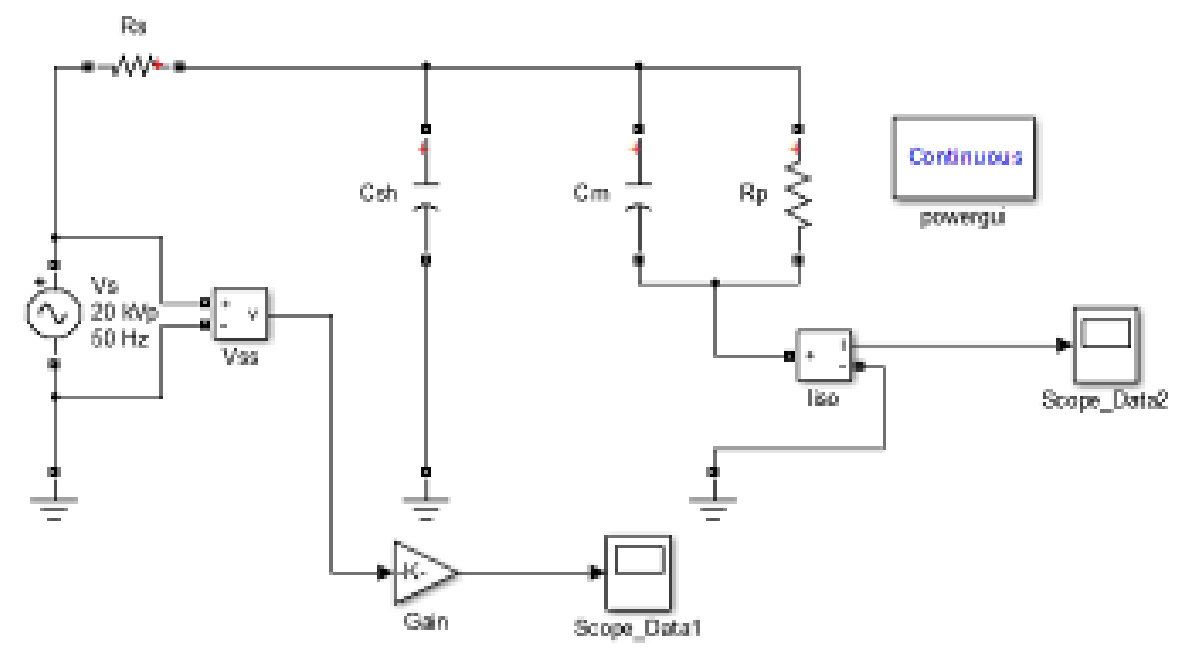

Figure 1. Test model with Matlab Simulink. 
The insulator specification that is used in this research is based on the previous research [5]. The 20 $\mathrm{kV}$ AC supply voltage component as the voltage applied to the test object (insulator) is equipped with an input resistance (Rs) for the formation of an impulse voltage. The test insulator model used is an equivalent series of suspension polymer insulators placed on the transmission line tower. The dimensions of the Polymeric Suspension type FXB-24/70 outdoor insulator as a test insulator.

In this research, the absolute permittivity $(\varepsilon)$ based on Shunt capacitance $\left(C_{s} h\right)$ that is determined from the cross-sectional dimensions (area and length) of the surface of the polymer insulator to the surface of the transmission tower and the relative permittivity of air, is equal to $8.854 \times 10^{-12} \mathrm{~F} / \mathrm{m}$. Due to the relative permittivity $\left(\varepsilon_{\mathrm{r}}\right)$ of cross-sectional dimensions (area and length) of polymer insulator material capacitance $\left(C_{m}\right)$ is 6 , therefore, the absolute permittivity $(\varepsilon)$ becomes $8,854 \times 10^{-12} \times 6 \mathrm{~F} / \mathrm{m}$. The pollution resistance on the surface of the insulator $\left(R_{p}\right)$ is determined based on the

conductivity of the pollution at a temperature of $20{ }^{\circ} \mathrm{C}$ in various levels of pollution severity (very light, mild, moderate, and severe). The leakage current crossing the surface of the insulator that is called as a leakage current signal is measured by the current measuring element. A scope device is connected from the current measuring element to read the leakage current measurement.

\section{RESULTS AND DISCUSSION}

Based on the cross-sectional area of the insulator $121.11 \mathrm{~cm}^{2}$; length of the insulator $28.5 \mathrm{~cm}$, and volume of solution $1000 \mathrm{~cm}^{3}$ resulted in insulator surface resistance to $\mathrm{NaCl}$ pollution. ESDD is determined by artificial pollution in the laboratory based on the IEC 60507 standard. The ESDD value is calculated using equation (10), the resulting ESDD still meets the range of typical values for pollution severity according to IEEE 4-1995 standards as shown in Table 1 .

Table 1. Surface resistance of insulators to salt pollution $(\mathrm{NaCl})$ and ESDD.

\begin{tabular}{|c|c|c|c|c|c|}
\hline No. & Severity & $\begin{array}{c}\sigma_{20} \\
(\mu \mathrm{S} / \mathrm{cm})\end{array}$ & $\begin{array}{c}\mathrm{Rp} \\
(\mathrm{M} \Omega)\end{array}$ & $\begin{array}{c}\mathrm{Sa} \\
\left(\mathrm{mg} / \mathrm{cm}^{3}\right)\end{array}$ & $\begin{array}{c}\mathrm{ESDD} \\
\left(\mathrm{mg} / \mathrm{cm}^{2}\right)\end{array}$ \\
\hline 1. & Very Light & 0.7989 & 0.2945 & 0.0031 & 0.0260 \\
\hline 2. & Light & 1.7907 & 0.1314 & 0.0072 & 0.0597 \\
\hline 3. & Moderate & 2.1594 & 0.1089 & 0.0087 & 0.0724 \\
\hline 4. & Heavy & 4.7368 & 0.0496 & 0.0196 & 0.1626 \\
\hline
\end{tabular}

Figure 2 shows the measurement results of the leakage current that crosses the surface of the insulator at a very light pollution level with a pollution resistance of $0.2945 \mathrm{M} \Omega$. The leakage current signal waves for two cycles are distorted with high frequency forming a whole in a positive direction with asymmetrical waveforms at all times. The distortion magnitude of the leakage current wave signal is very large, exceeding the magnitude of the fundamental leakage current signal of $1.128 \mathrm{~mA}$ with a phase angle of $0.55^{\circ}$.

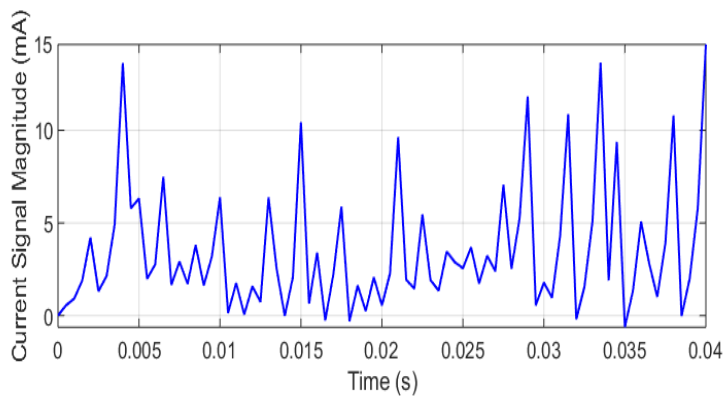

Figure 2 The leakage current for very light polluted insulators.

The frequency spectrum of the leakage current signal for very light polluted insulators is shown in Figure 3. The harmonic leakage current signal produced with the average current magnitude at odd harmonic frequencies is greater than the even harmonic frequency, so that the leakage current wave signal is in a positive position. THD of the harmonic frequency of the leakage current on the insulator is very light polluted at $360.15 \%$.

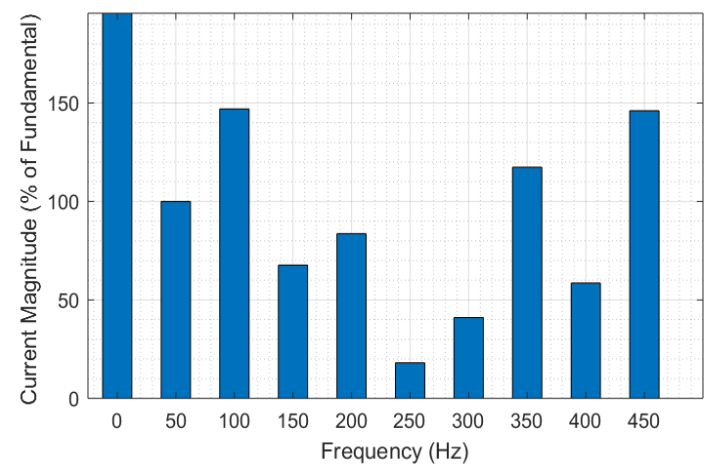

Figure 3 Harmonic frequency spectrum in very light polluted insulators.

The results of the measurement of the leakage current that crosses the surface of the insulator at a light pollution level with a pollution resistance of $0.1314 \mathrm{M} \Omega$ are shown in Figure 4. The leakage current signal waves formed for two distorted cycles with high frequency are almost entirely positive with asymmetrical waveforms at all times. The distortion magnitude of the leakage current wave signal is slightly 
smaller than the fundamental leakage current signal magnitude of $1.893 \mathrm{~mA}$ with a phase angle of $0.25^{\circ}$.

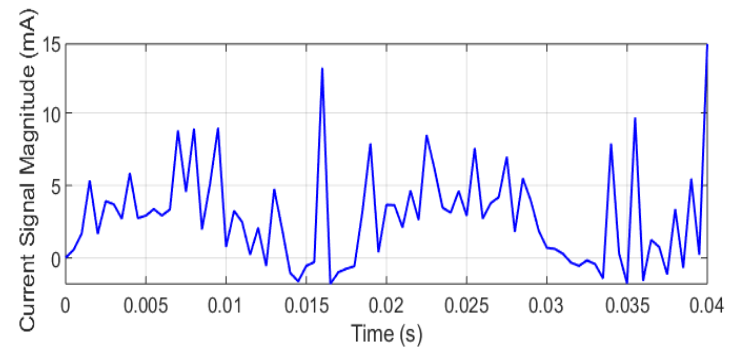

Figure 4 Leakage current for light polluted insulators.

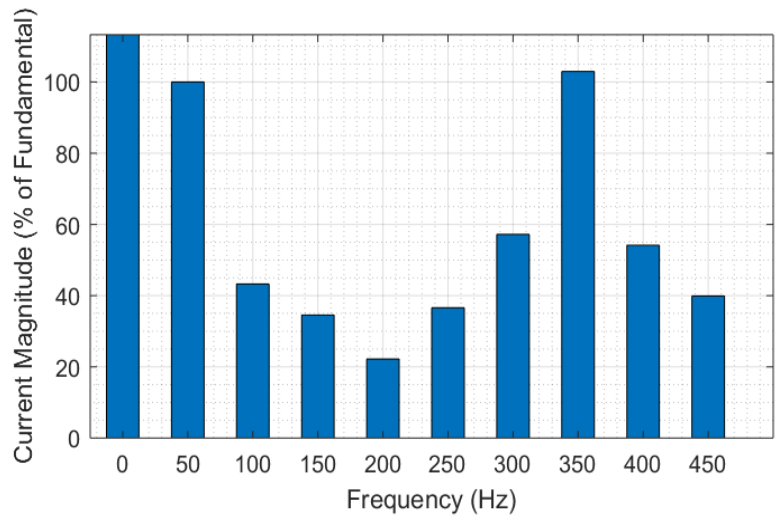

Figure 5 Harmonic frequency spectrum in light polluted insulators.

Figure 5 shows the frequency spectrum of the leakage current signal for a lightly polluted insulator. The harmonic leakage current signal generated with the average current magnitude at odd harmonic frequency is greater than even harmonic frequency, so that the leakage current wave signal is almost entirely in a positive position. THD of the harmonic frequency of the leakage current in lightly polluted insulators is $230.96 \%$.

Figure 6 shows the measurement results of the leakage current that crosses the surface of the insulator at a moderate pollution level with a pollution resistance of $0.1089 \mathrm{M} \Omega$. The leakage current signal waveform for two cycles is distorted with high frequency forming a slightly negative direction with asymmetric waveforms at all times. The distortion magnitude of the leakage current wave signal decreases compared to the previous one and is smaller than the fundamental leakage current signal magnitude of 3.206 $\mathrm{mA}$ with a phase angle of $0.20^{\circ}$.

The frequency spectrum of the leakage current signal for a moderately polluted isolator is shown in Figure 7. The resulting harmonic leakage current signal with the average current magnitude at odd harmonic frequencies is greater than the even harmonic frequency, so that the current wave signal is mostly leaking in a positive position. THD of the harmonic frequency of the leakage current in the insulator with moderate pollution is $133.20 \%$.

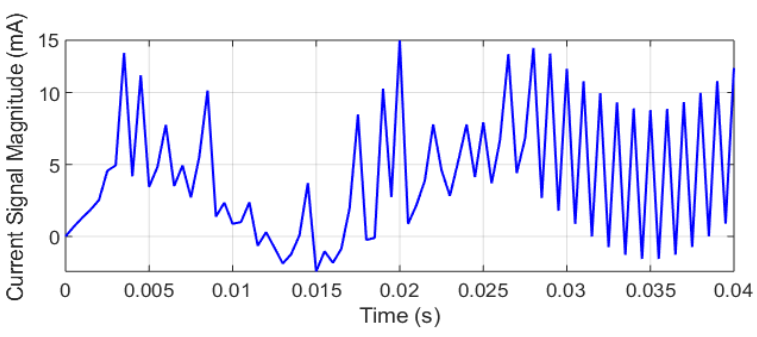

Figure 6 The leakage current for moderate polluted insulators.

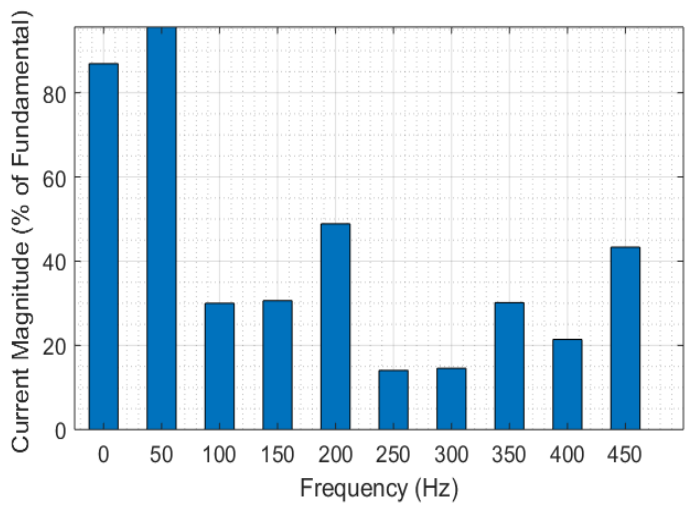

Figure 7 Harmonic frequencies spectrum in moderate polluted insulators.

The results of the measurement of the leakage current that crosses the surface of the insulator at a heavy pollution level with a pollution resistance of $0.0496 \mathrm{M} \Omega$ are shown in Figure 8. The leakage current signal waves formed for two high frequency distorted cycles are almost partially in the positive direction and some are in the negative with an almost symmetrical waveform at all times. The distortion magnitude of the leakage current wave signal is much smaller than the fundamental leakage current signal magnitude of 6.714 $\mathrm{mA}$ with a phase angle of $0.09^{\circ}$.

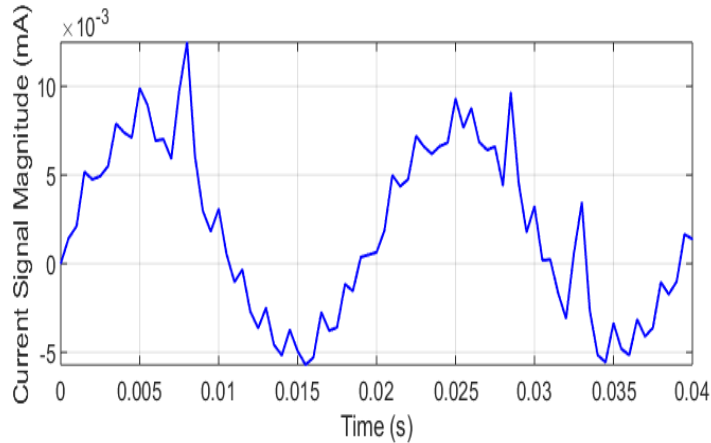

Figure 8 Leakage currents for heavily polluted insulators. 


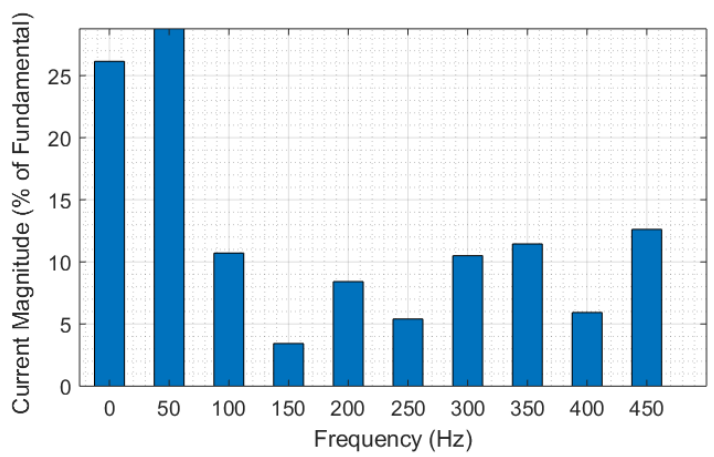

Figure 9 Harmonic frequencies spectrum in heavily polluted insulators.

The frequency spectrum of the leakage current signal for heavily polluted insulators is shown in Figure 9. The resulting harmonic leakage current signal with the mean current magnitude at odd harmonic frequencies is almost the same as the even harmonic frequency magnitude, so that the leakage current wave signal is $40 \%$ in a negative position. which forms a slightly distorted sine wave. THD of the harmonic frequency of the leakage current in the insulator with moderate pollution is $30.76 \%$.

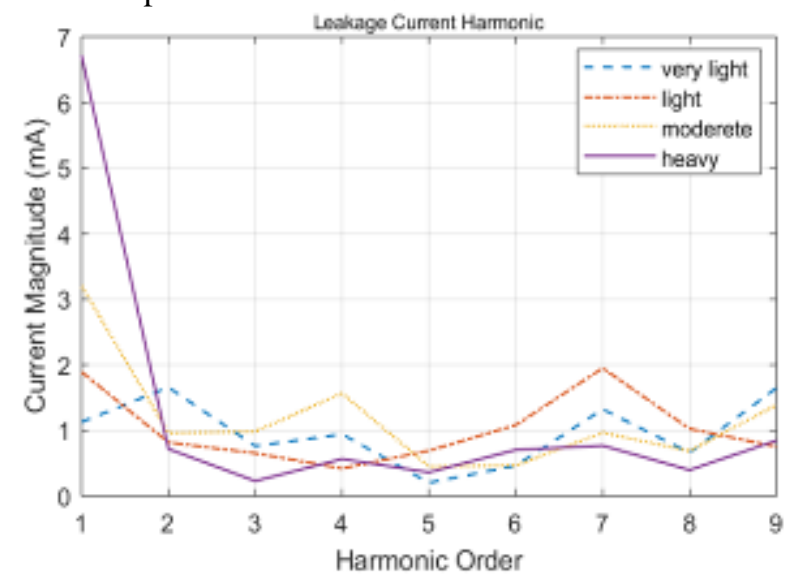

Figure 10 Harmonic leakage current for pollution severity in insulators.

Figure 10 illustrates the harmonic leakage current for the pollution severity on the surface of the insulator where the greater the magnitude of the leakage current, the smaller the wave distortion for each harmonic order. Isolators with very light pollution levels produce a leakage current magnitude of $1.128 \mathrm{~mA}$ with a THD of $360.15 \%$, while heavy pollution levels produce a leakage current magnitude of $6,714 \mathrm{~mA}$ with a THD of $30.76 \%$. This condition indicates that the greater the magnitude of the leakage current for the severity of the heavy pollution, but the smaller the harmonic distortion with the waveform of the leakage current signal leading to a sinusoidal waveform.

\section{CONCLUSION}

Based on the results of measurement and discussion, it can be concluded as follows:

1. The leakage current that occurs when the surface of the insulator is very lightly polluted with a small magnitude has an unsymmetrical waveform that is distorted and leads to a positive with a large THD.

2. The magnitude of the leakage current with the surface of the insulator is polluted, which leads to greater weight, but the harmonic distortion and THD are getting smaller with the waveform of the leakage current signal leading to a sinusoidal waveform.

\section{SUGGESTIONS}

This research can be continued through measurements by making artificial conditions of environmental stresses such as temperature, relative humidity, and condensation which can affect the conductivity of pollution on the surface of the insulator.

\section{REFERENCES}

[1] Amin, Mohammad, Mohammad Akbar, and Salman Amin. "Hydrophobicity of silicone rubber used for outdoor insulation (an overview)." Rev. Adv. Mater. Sci 16 (2007): 10-26.

[2] Manjang, Salama, and Masayuki Nagao. "Characteristics of high voltage polymer insulator under accelerated artificial tropical climate multi stress aging." In Proceedings of 2011 International Symposium on Electrical Insulating Materials, pp. 221-224. IEEE, 2011.

[3] Mekala, K., S. Chandrasekar, and R. Samson Ravindran. "Investigations of Accelerated Aged Polymeric Insulators Using Partial Discharge Signal Measurement and Analysis." Journal of Electrical Engineering \& Technology 10, no. 1 (2015): 299-307.

[4] Hussain, M. Majid, Shahab Farokhi, S. G. McMeekin, and Masoud Farzaneh. "The effects of salt contamination deposition on $\mathrm{HV}$ insulators under environmental stresses." In 2015 IEEE 11th International Conference on the Properties and Applications of Dielectric Materials (ICPADM), pp. 616-619. IEEE, 2015.

[5] Sitompul, Carlos, Zainuddin Nawawi, and Irfan Jambak. "Detection of Polymer Insulator Surface Based on the Effects of Tropical Climate Using Partial Discharge Signals Measurement." In Journal of Physics: Conference Series, vol. 1500, p. 012019. 2020. 